Rapport - Société canadienne d'histoire de l'Église catholique

\title{
Le martyre blanc des missionnaires du Saguenay
}

\section{Victor Tremblay}

Volume 31, 1964

URI : https://id.erudit.org/iderudit/1007345ar

DOI : https://doi.org/10.7202/1007345ar

Aller au sommaire du numéro

Éditeur(s)

La Société canadienne d'histoire de l'Église catholique

ISSN

0318-6148 (imprimé)

1927-7075 (numérique)

Découvrir la revue

Citer cet article

Tremblay, V. (1964). Le martyre blanc des missionnaires du Saguenay. Rapport

- Société canadienne d'histoire de l'Église catholique, 31, 45-53.

https://doi.org/10.7202/1007345ar

Tous droits réservés @ La Société canadienne d'histoire de l'Église catholique, 1965
Ce document est protégé par la loi sur le droit d'auteur. L’utilisation des services d'Érudit (y compris la reproduction) est assujettie à sa politique d'utilisation que vous pouvez consulter en ligne.

https://apropos.erudit.org/fr/usagers/politique-dutilisation/ 


\title{
Le martyre blanc des missionnaires du Saguenay
}

\author{
Il s'agit des conditions particulièrement pénibles que les \\ missionnaires de cette partie du Canada étaient obligés de subir \\ dans l'exercice de leur apostolat, conditions qui faisaient de leur \\ vie un long et inévitable martyre.
}

La page d'histoire que je présente aujourd'hui peut faire suite à celle que j'ai communiquée au congrès de 1946 sous le titre : "L'évangélisation du Saguenay par les Jésuites. » Elle a pour but de faire connaître un aspect généralement ignoré de cette œuvre apostolique : les conditions particulières qui rendaient extraordinairement pénibles la vie et le travail des missionnaires dans ce champ d'activité et qui en faisaient un véritable martyre sans effusion de sang, ce que j'appelle "le martyre blanc».

Le pays du Saguenay, souvent appelé « Royaume du Saguenay », comme on le désignait à l'origine, est un vaste territoire qui occupe la partie nord des régions colonisées de la province de Québec. Sa position, fixée en 1732 par l'autorité royale de France et reconnue en 1926 devant le Conseil Privé d'Angleterre, s'étendait de l'est à l'ouest depuis la rivière Moisie, en bas de Sept-Iles, jusqu'à l'Abitibi, et du sud au nord à partir des montagnes qui ferment l'horizon de la ville de Québec jusqu'aux solitudes de l'Ungava, au-delà du grand lac Mistassini. Sa superficie équivaut à cinq fois celle de la province du Nouveau-Brunswick.

Vaste pénéplaine de montagnes aux sommets arrondis, dans laquelle est enchâssée la large vallée du lac Saint-Jean, et coupée par la rivière et le fjord Saguenay, qui est le débouché du lac vers le fleuve SaintLaurent, ce pays, au temps des missions indiennes, était tout couvert par un manteau de forêt qui servait d'habitat à de nombreux animaux à fourrures et à des tribus clairsemées, à l'état nomade, vivant uniquement de chasse et de pêche. Un merveilleux réseau de rivières et de lacs fournissait à ces peuples des voies de communication.

Ces peuples, de race algonquine, étaient désignés sous le nom général de « Montagnais». La nécessité de tirer leur subsistance de la chasse et de la pêche les forçait à se disperser par familles et à se partager les territoires entre elles, conséquemment à vivre isolés, excepté pendant deux mois l'été, du milieu de juin au milieu d'août, alors qu'avaient lieu les rencontres, pour la traite des fourrures, avec les commerçants européens, à certains endroits déterminés. Ces endroits étaient principalement Tadoussac, à l'entrée du fjord Saguenay, Chicoutimi, à la tête de la navigation maritime sur le fjord, et Métabetchouan, sur le bord du lac Saint-Jean. 
Il faut signaler que ce territoire était alors un pays fermé. Géographiquement isolé par la large chaîne montagneuse des Laurentides, avec une seule entrée naturelle, le fjord, ce qui le rendait facile à garder, le territoire du Saguenay était de plus réservé, à titre de Domaine du Roi, pour la traite des fourrures. Seuls les agents de ce commerce et les missionnaires étaient autorisés à y pénétrer.

Tel était le champ d'apostolat des missionnaires des Montagnais.

Cet apostolat couvre une période longue de cent soixante-sept ans, dont cent quarante et un ans à la charge des Jésuites, soit six fois la durée de leurs missions au pays des Hurons. Après les timides essais tentés par les Récollets entre 1615 et 1622 et plus tard par le père Paul Le Jeune, jésuite, à titre d'expérience, en 1634, l'œuvre d'évangélisation chez les Montagnais du Saguenay commença effectivement à Tadoussac en 1641 et finit avec la mort du dernier missionnaire jésuite, le père Jean-Baptiste de La Brosse, en 1782. Pendant cette période, vingt et un missionnaires participèrent à la tâche, quelques-uns pendant peu de temps, d'autres pendant une partie notable de leur carrière, d'autres à longueur de vie, comme le père François de Crespieul, qui y a consacré trente et un ans et qui y est mort, à 64 ans d'âge.

Le premier qui tenta l'expérience de l'apostolat chez les Montagnais est le récollet Jean Dolbeau. A l'automne de 1615, il s'associa à une famille de Tadoussac et entreprit de la suivre dans ses migrations, tant pour apprendre la langue que pour se renseigner sur la manière de vivre. Il avait choisi pour cela la saison d'hiver, où l'on est moins exposé à manquer de nourriture, le gibier étant plus facile à atteindre en cette saison que pendant l'été.

Le pauvre religieux supporta courageusement l'épreuve du froid dans les courses en raquettes ou sous la tente d'écorce, où on touche la glace d'un côté pendant que de l'autre on grille au feu; il s'accommoda de la pénible promiscuité de tout ce monde, hommes, femmes, enfants et chiens, entassé dans l'étroit abri de la tente pendant les longues nuits d'hiver et une grande partie des jours; il se résigna à la malpropreté inévitable et sans nom de ces gens et de ce genre de vie et il accepta la nourriture répugnante, sans apprêts et sans sel, que ses hôtes lui offraient. Vainqueur de toutes les incommodités et de toutes les misères, il fut vaincu par une à laquelle on ne penserait pas à moins d'y être initié. "Ayant, par la grâce de Dieu, surmonté toutes les autres difficultés qui se rencontrent en semblables occasions, écrit le frère Sagard, la fumée, qui est en grande abondance dans leurs cabanes, notamment lorsqu'il fait un temps nébuleux et de neige, lui pensa perdre la vue...; il fut plusieurs jours sans pouvoir ouvrir les yeux, qui lui faisaient une douleur extrême, tellement que dans l'appréhension 
que son mal augmentât il fut contraint de les quitter après deux mois de temps et de revenir à l'habitation....

Sagard relate aussi les douloureuses expériences du père Irénée Piat et du père Joseph Le Caron dans les voyages qu'ils entreprirent avec les Montagnais vers Tadoussac.

C'est la hauteur des neiges : "cinq ou six pieds », qui condamne à de longs trajets à la raquette; c'est la pluie glacée, qui rend la marche quasi impossible avec les raquettes, dont on ne peut pas se passer; ce sont les brusques changements de température, les tempêtes et les froids mordants; c'est le campement à la belle étoile où " le ciel était sa couverture et la terre son lit mollet "; c'est le manque quasi total de nourriture, impossible à apporter en voyage ou dévorée dès le départ par les Indiens accoutumés à manger tout de suite ce qu'ils ont, quittes à jeûner jusqu'à la prochaine possibilité d'en trouver; c'est la vitesse de la marche, que l'Indien ne sait pas accommoder à la capacité du religieux peu entraîné à cet exercice; ce sont les portages dans la boue et la neige en passant du fleuve à la terre, que le mauvais temps force à regagner promptement; c'est l'épuisement jusqu'à l'extrême limite, au point de tomber là pour y mourir à moins d'un secours providentiel. Lors de sa première aventure de ce côté le père Piat, qui avait su tout supporter, fut réduit à abandonner la partie devant le spectacle de la sorcellerie infernale qu'on appelle le ouabano.

Des misères de ce genre se rencontraient, mais à un degré beaucoup moindre, dans les autres missions plus favorisées par le climat. Le froid est déjà à lui seul un élément de souffrance plus rigoureux et plus prolongé en ces lieux qu'ailleurs, et pour s'en protéger on n'a que l'abri insuffisant et précaire de la tente d'écorce de bouleau, qui n'arrête que partiellement le vent et les pluies glacées.

Après son expérience de l'hivernement avec les Montagnais (1634), le père Paul Le Jeune, jésuite, décrit en détail les inconvénients du séjour sous la tente indienne en hiver, cette petite cabane légère qu'on transporte au gré du besoin, sans fenêtre et si étroite qu'on y est entassé les uns sur les autres, et cela presque à longueur de jour comme de nuit pendant toute la saison froide, qui dure près de sept mois. J'ai choisi cette description qui, à part quelques détails particuliers, résume toutes les autres et qui fait autorité.

"Vous ne sauriez tenir debout dans cette maison, écrit-il, tant pour sa bassesse que pour la fumée qui suffoquerait, et par conséquent il faut être toujours couché ou assis sur la plate terre; c'est la position ordinaire des Sauvages. De sortir dehors, le froid, la neige, le danger de s'égarer dans ces grands bois vous font rentrer plus vite que le vent et vous tiennent en prison dans un cachot qui n'a ni clef ni serrure.

"Ce cachot, outre la fâcheuse posture qu'il faut y tenir sur un lit de terre, a quatre grandes incommodités : le froid, le chaud, la fumée et les chiens. Pour le froid, vous avez la tête à la neige, il n'y a qu'une branche de pin entre deux, bien souvent rien que votre bonnet; les vents 
ont liberté d'entrer par mille endroits; car ne vous figurez pas que ces écorces sont jointes comme un papier collé sur un châssis, elles ressemblent bien souvent à l'herbe à mille pertuis, sinon que leurs trous et leurs ouvertures sont un peu plus grands. Et quand il n'y aurait que l'ouverture d'en haut, qui sert de fenêtre et de cheminée tout ensemble, le plus gros hiver de France y pourrait tous les jours passer tout entier sans empressement...

"Cependant le froid ne m'a pas tant tourmenté que la chaleur du feu. Ũn petit lieu comme sont leurs cabanes s’échaữe aisément par un bon feu, qui me rôtissait parfois et me grillait de tous côtés, à raison que, la cabane étant trop étroite, je ne savais (pas) comment me défendre de son ardeur. D'aller à droite ou à gauche, vous ne sauriez, car les Sauvages qui vous sont voisins occupent vos côtés; de reculer en arrière, vous rencontrez cette muraille de neige ou les écorces de la cabane qui vous bornent. Je ne savais en quelle posture me mettre; de m'étendre, la place était si étroite que mes jambes eussent été à moitié dans le feu; de me tenir en peloton et toujours raccourci comme ils font, je ne pouvais pas aussi longtemps qu'eux. Mes habits ont été tout rôtis et brûlés...

"Pour les chiens, que j'ai dit être l'une des incommodités des maisons des Sauvages, je ne sais si je les dois blâmer ... Ces pauvres bêtes, ne pouvant résister à l'air hors de la cabane, venaient se coucher tantôt sur mes épaules, tantôt sur mes pieds, et comme je n'avais qu'une simple catalogne pour me servir de matelas et de couverture tout ensemble, je n'étais pas marri de cet abri, leur rendant volontiers une partie de la chaleur que je tirais d'eux. Il est vrai que, comme ils étaient grands et en grand nombre, ils me pressaient parfois et m'importunaient si fort, qu'en me donnant un peu de chaleur ils me dérobaient tout mon sommeil... Ces animaux, affamés d'autant qu'ils n'avaient pas plus que nous de quoi manger, ne faisaient qu'aller et venir, rôder partout dans la cabane ...; ils nous passaient souvent et sur la face et sur le ventre... avec une telle importunité qu'étant las de crier et de les chasser je me couvrais quelquefois la face et je leur donnais liberté de passer par où ils voudraient...»

Ainsi coincé entre la muraille glacée de la tente ou de la neige et le feu qui le grille, piétiné par les chiens qui lui prêtaient leurs odeurs et leurs puces, le missionnaire trouvait encore ces inconvénients tolérables, "mais pour la fumée, écrit-il, je vous confesse que c'est un martyre. Elle me tuait et me faisait pleurer incessamment...; elle nous terrassait parfois tous...; il fallait mettre la bouche contre terre pour pouvoir respirer; encore que les Sauvages soient accoutumés à ce tourment..., ils étaient contraints aussi bien que moi de se coucher sur le ventre et de manger quasi la terre pour ne pas boire la fumée. J'ai quelquefois demeuré plusieurs heures en cette situation, notamment dans les plus grands froids et lorsqu'il neigeait, car c'était en ces tempslà que la fumée nous assaillait avec plus de fureur, nous saisissant à la gorge, aux narines et aux yeux... J'ai cru plusieurs fois que j'al- 
lais être aveugle; les yeux me cuisaient comme du feu, ils me pleuraient ou distillaient comme un alambic et je ne voyais plus que confusément...»

Le Père ajoute ce détail qui complète le tableau : \& Quelqu'un me dira que je devais sortir de ce trou enfumé et prendre l'air; je lui répondrai que l'air était ordinairement en ce temps-là si froid que les arbres, qui ont la peau plus dure que celle de l'homme et le corps plus solide, ne pouvaient lui résister, se fendant jusques au cœur (en) faisant un bruit comme celui d'un mousquet en s'éclatant. Je sortais néanmoins de cette tannière, fuyant la rage de la fumée pour me mettre à la merci du froid, contre lequel je tâchais de m'armer, m'enveloppant de ma couverture comme un Irlandais, et en cet équipage, assis sur la neige ou quelque arbre abattu, je récitais mes heures (de bréviaire)...

Merci au père Le Jeune de nous avoir ainsi décrit le supplice de la vie sous la tente d'écorce en hiver au pays du Saguenay. Il n'est pas seul à l'avoir connu et d'autres à sa suite en ont révélé des aspects, mais il le fait avec la précision d'un rapporteur conscient du devoir de renseigner exactement, et cela donne un poids particulier à son témoignage.

On pourrait lui emprunter aussi la description de la nourriture. - Au commencement que je fus avec eux, comme ils ne salent ni leurs bouillons ni leurs viandes et que la saleté même fait leur cuisine, je ne pouvais (pas) manger de leur salmigondis; je me contentais d'un peu de galette et d'un peu d'anguille boucanée...; je m'affamai devant que la famine nous accueillit. Cependant nos Sauvages faisaient tous les jours des festins, en sorte que nous nous vîmes en peu de temps sans pain, sans farine et sans anguille, et sans aucun moyen d'être secourus... Le peu que donnait la chasse "servait plutôt pour ne point mourir que pour vivre ... Pour l'ordinaire nous mangions une fois en deux jours... Sur la fin de nos vivres, quand je pouvais avoir une peau d'anguille pour ma journée je me tenais pour bien déjeuner, bien dîner et bien souper. Au commencement je m'étais servi d'une de ces peaux pour réparer une soutane de toile que j'avais sur moi, ayant oublié d'apporter des pièces; mais voyant que la faim me pressait si fort, je mangeai mes pièces, et si ma soutane eut été de même étoffe, je vous réponds que je l'eusse rapportée bien courte à la maison. Je mangeais bien les vieilles peaux d'orignal, qui sont bien plus dures que les peaux d'anguille; j'allais dans le bois brouter le bout (des branches) des arbres et ronger les écorces plus tendres. »

Un sujet de souffrance qu'on ne penserait même pas à soupçonner, c'est le manque d'eau potable. Le missionnaire en décrit le supplice, alors que malade il demanda de l'eau sans pouvoir obtenir autre chose que de la "neige fondue dans une chaudière dont le cuivre était moins épais que la saleté ", et il nous met au défi d'en faire l'expérience : " Qui voudra savoir l'amertume de ce breuvage, écrit-il, qu'il le tire d'un vaisseau sortant de la fumée et qu'il en goûte." Il ajoute cette 
observation : "Une âme bien altérée de la soif des souffrances trouverait ici de quoi se rassasier.»

Ce n'est pas encore tout. "Pour faire entièrement connaître ce qu'on peut souffrir avec ce peuple, écrit le religieux, il me reste à parler de leur conversation. " Il s'agit ici du compagnonnage d'un sorcier qu'il dut subir malgré les promesses contraires qu'on lui avait faites. "Ce misérable homme et la fumée m'ont été les plus grands tourments que j'ai endurés parmi ces barbares; ni le froid, ni le chaud, ni l'in-

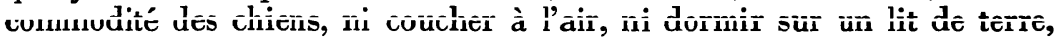
ni la posture qu'il faut toujours tenir dans leurs cabanes...., ni la faim, ni la soif, ni la pauvreté et saleté de leur boucan, ni la maladie : tout cela ne m'a semblé que jeu en comparaison de la fumée et de la malice du sorcier...."

J'ai voulu citer d'assez longs extraits de la relation de 1634 du père Le Jeune, parce que précisément elle est le rapport d'une expérience tentée par le vaillant religieux expressément pour connaître les conditions de vie chez les Montagnais, en vue de savoir s'il y avait possibilité pour un missionnaire de les supporter. Sa conclusion est péremptoire : il est convaincu que la chose est impossible. "De vouloir les suivre, écrit-il, il faudrait autant de religieux qu'ils ont de cabanes; encore n'en viendrait-on pas à bout, car ils sont tellement occupés à quêter leur vie parmi ces bois, qu'ils n'ont pas le loisir de se sauver... Je ne crois point que de cent religieux il y en ait dix qui puissent résister aux travaux qu'il faudrait endurer à leur suite.»

Quand on se rappelle que c'est le jésuite Paul Le Jeune qui écrit cela, en 1634, et pour publication dans les Relations, on est obligé de prendre l'observation à la lettre. Et même, après la lecture de tous les détails de ce terrible hiver, des péripéties dramatiques du retour, on est convaincu qu'il n'exagère en rien et qu'on devrait peut-être réduire à la proportion de deux sur cent le nombre des missionnaires capables de supporter un tel genre de vie.

Pourtant le père Le Jeune n'a pas encore tout dit. Il a sans doute expérimenté la situation tragique d'un petit groupe d'affamés isolé de tout dans les bois, le pénible métier des déménagements répétés du campement en hiver par n'importe quel temps - la chose s'était produite vingt-trois fois au cours de l'hivernement - le martyre des nuits à la belle étoile dans la neige ou sous la pluie, le supplice du froid sans possibilité de se réchauffer, supplice qui faisait dire à un missionnaire de notre époque : " Le fait d'avoir mis le feu au lieu du froid dans l'enfer est une preuve de la miséricorde de Dieu jusque dans le châtiment des damnés »; il avait subi les péripéties énervantes et les périls de la navigation en frêle canot d'écorce dans les rapides, parmi les écueils de toutes sortes, les glaces et les bourrasques, la douloureuse corvée des portages, pour ne parler que des inconvénients de la vie hors de la tente étroite et enfumée; il lui a manqué de connaître les courses à longue distance qu'imposa aux autres missionnaires venus après lui 
l'exercice de leur ministère dans l'immense pays saguenéen, cinq fois grand comme la province du Nouveau-Brunswick.

Il faudrait suivre, par exemple, l'apostolat d'un père Jean Dequen, d'un père Druillettes, d'un père Albanel, d'un père de Crespieul, d'un père Favre, d'un père Nouvel, d'un père Laure, d'un père Maurice, d'un père Coquart, d'un père de La Brosse. On ne compte pas les hivernements de ces missionnaires et leurs longues courses apostoliques. Signalons quelques faits à titre d'exemples.

En 1650, les Indiens du Saguenay " ont mené le père Gabriel Druillettes dans ces contrées par un chemin nouveau mais très affreux, afin qu'il visitât et consolât ceux qui ne pouvaient (pas) venir le trouver à Tadoussac ». Le père Jean Dequen avait fait de même, en vitesse, trois ans auparavant en se rendant au lac Saint-Jean, distance de 120 milles; il y retournera deux fois et deux fois il se rendra chez les Oumamiouek de la Côte Nord, "à 80 lieues de Tadoussac ». En 1661, les pères Druillettes et Dablon se rendent à Nekoubau, à 250 milles de Tadoussac, au prix de nombreux et pénibles portages. Tout le monde connaît les fameux voyages du père Albanel jusqu'à la baie d'Hudson de 1671 à 1674; tout le monde ne sait pas que ce missionnaire fut pendant vingtdeux ans à la tâche chez les Montagnais, qu'il hiverna nombre de fois avec eux et qu'il y fut un merveilleux apôtre. Dans le premier voyage à la baie d'Hudson « il a souffert tout ce qu'on peut s'imaginer », dit la relation de 1679. Il écrit lui-même à ce sujet : "Je puis assurer que de dix hivernements que j'ai faits dans les bois avec les Sauvages, les neuf premiers ne m'ont pas tant donné de peine que ce dernier."

Il en avait pourtant connu de dures. Dans sa première lettre au père Le Mercier, au printemps de 1670, il écrivait : " ... Pendant ces huit mois que j'ai passés dans des continuelles et précieuses occasions de souffrir, ce n'est pas néanmoins la rigueur excessive des saisons, ni l'extrême fatigue des chemins, ni la nécessité des vivres qui m'ont donné le plus de peine; je sais que nos Pères qui passent l'hiver dans les forêts souffrent toutes ces incommodités; mais rien ne m'a été plus sensible que la vue des misères incroyables et de l'abandon où étaient réduits nos pauvres Sauvages... Je vous avoue que mon cœur en était si sensiblement touché, que je mets cette peine au nombre des plus rudes que j'aie jamais ressenties." Il aurait pu ajouter : "et partagées ». Les Indiens avaient été victimes de la petite vérole, qui avait enlevé "environ 250 personnes ". Après avoir couru au secours des pestiférés jusqu'à l'île Verte, il revint à son poste principal "qui pouvait passer pour un hôpital de malades", et pendant le mois de janvier "le plus fort de mon exercice, écrit-il, a été de secourir les malades, d'exhorter les mourants et d'ensevelir les morts ... J'aurais pu pratiquer de grands actes de vertu et surtout d'une mortification qui n'est pas petite, me trouvant obligé de demeurer dans un lieu infecté d'une puanteur horrible». Et comme complément à tout cela il eut sa part de maladie. "J'avais toute la tête furieusement enflée et le visage couvert de pustules comme de petite vérole; une grande douleur d'oreille 
me prit, avec un furieux mal de dents; mes lèvres devinrent comme mortes et mes yeux furent extrêmement incommodés d'une fluxion. Pour comble de tous ces maux, j'avais une très grande difficulté de respirer. » Il en avait assez pour expirer; une promesse à saint FrançoisXavier lui valut de s'en tirer indemne, et après un mois de ministère actif à Tadoussac il s'embarquait pour visiter les lointaines missions de la Côte jusqu'à la Pointe des Monts, à 150 milles de Tadoussac.

On a l'embarras du choix quant aux exemples de longues courses âpostôliquies dañs l'immonsc pays du Sagucnay. Voici un cas: les courses du père François de Crespieul en 1673-1674. Après un hivernement dans les forêts du lac Saint-Jean, on le trouve à Chicoutimi, à 100 milles de distance, pour une partie de l'été. De là il va donner une mission aux Ilets de Jérémis, sur la côte du fleuve, trajet de 140 milles. De retour à Chicoutimi, il monte dans le Nord hiverner aux sources de la rivière Shipshaw, 80 à 90 milles. En janvier 1674, averti que le père Albanel est arrêté par la maladie, il vole à son secours à travers les bois et faillit périr sur la glace du grand lac Pomouscachiou dans une tempête. En mars, poursuivi par les Iroquois, qui sont rendus là, il descend au lac Saint-Jean, 80 milles, puis remonte au grand lac Mistassini, 180 milles, au secours d'un groupe de néophytes; il en baptise cent deux. De retour à Chicoutimi à la fin de mai, il part pour Québec avec une délégation de Montagnais qu'il doit présenter au gouverneur. Et ça continue ainsi. Au printemps suivant il est chez les Papinachois de la Côte, le 12 juillet il est à la mission de Nekoubau, à 450 milles de celle des Papinachois.

Et si on veut savoir dans quelles conditions il fallait souvent accomplir ces courses, en voici une petite description. Le père de Crespieul est cette fois appelé pour un malade, à la fin de l'automne de 1677. a Je me suis mis en chemin pour cela le 24 , de novembre. Il fallut marcher toute la journée, ayant la neige jusqu'aux genoux, gravir des montagnes très difficiles, passer des lacs sans craindre la froidure des eaux dans lesquelles nous marchions, traverser des bois épais qui nous déchiraient le visage et les habits, sauter d'arbre en arbre pour se tirer de certains endroits où le vent les a culbutés les uns sur les autres. Cependant, les forces me manquant le soir, il nous fallut jeter des branches de sapin sur la neige pour nous y coucher et passer la nuit, sans abri et sans écorces pour nous couvrir contre la neige qui nous mouillait et nous gelait en même temps. Toute la nuit se passa à prendre patience du mieux que nous pûmes; au point du jour je partis à jeûn, croyant avoir assez de forces pour gagner la cabane du malade et y dire la messe. Mais la longueur et la difficulté du chemin, dont les arbres embarrassaient le passage, me causèrent plusieurs blessures aux jambes, et m'ayant entièrement épuisé, je fus obligé de prendre un peu de thériaque, qui me donna assez de vigueur pour arriver à la cabane sur les deux heures après midi. " Le retour ne fut pas moins pénible. Un mois plus tard le Père fut tout près de mourir d'une maladie « occasionnée par les travaux et les fatigues qu'il est obligé d'endurer pour le salut de tous ces peuples errants ». 
Il en est ainsi des autres missionnaires qui, pendant cent quarante et un ans, se succédèrent dans ce vaste territoire, agrandi encore lorsque, après la conquête, le nombre réduit des religieux obligea le missionnaire du Saguenay de visiter en plus les Indiens de la baie des Chaleurs et du fleuve Saint-Jean. Leur principal centre était, selon les époques, Métabetchouan, au lac Saint-Jean, Chicoutimi, Tadoussac, mais les limites étaient d'un côté le lac Mistassini, et même Nemiskau, à michemin entre ce lac et la baie James, de l'autre Sept-Iles et la rivière Moisie. Entre les deux extrêmes une distance de plus de 900 milles, et pour la partie la plus régulièrement visitée, au rythme de deux, trois ou quatre fois par année, un trajet de 630 milles, redoublé par les retours.

A part les missions régulières, il fallait répondre à l'appel des besoins, soit aux divers postes de mission soit dans les bois, et les dangers de périr ne manquaient pas, spécialement dans les voyages précipités qu'il fallait faire sans en choisir le moment ni la route la moins difficile.

Tout cela en plus des inconvénients soufferts dans tous les milieux indiens : contacts pénibles, continuels, obligatoires, dont on pouvait se soulager quelquefois dans les autres pays de mission mais pas au Saguenay, oppositions suscitées par les divergences de vues et surtout par les sorciers, les dépravés et les apostats.

Pour terminer cette enquête, qu'on pourrait prolonger beaucoup, on devrait citer le témoignage du héros le plus authentique de cette inconcevable aventure, le père François de Crespieul. Le 21 avril 1647, alors qu'il achevait son " $26^{\circ}$ hivernement à l'emploi de la mission de Tadoussac ", il traçait de sa main, "pour l'instruction et l'édification * de ses successeurs, un tableau de " la vie d'un missionnaire montagnais ». Il y ramasse les misères que nous venons de décrire, dont il encadre l'énumération entre deux phrases lapidaires : "La vie d'un missionnaire montagnais est un long et lent martyre, est un exercice presque continuel de patience et de mortification et une vie vraiment pénitente et humiliante, surtout dans les cabanes et dans les chemins avec les Sauvages. » «a souffrance et la misère sont les apanages de ces saintes et pénibles missions. "

Il me semble qu'en conclusion de cette étude il n'est pas outré d'appeler martyre - martyre blanc si l'on veut, mais martyre véritable - la vie apostolique des anciens missionnaires du Saguenay.

\section{Victor TREMbLaY, P.D.}

Séminaire de Chicoutimi

Président de la Société Historique du Saguenay 